\title{
A novel group of HPV-related adenocarcinomas of the lower anogenital tract (vagina, vulva, and anorectum) in women and men resembling HPV-related endocervical adenocarcinomas
}

\author{
Lysandra Voltaggio $^{1} \cdot$ W. Glenn McCluggage ${ }^{2} \cdot$ Jeffrey S. Iding $^{3} \cdot$ Brock Martin $^{4} \cdot$ Teri A. Longacre ${ }^{4}$ \\ Brigitte M. Ronnett ${ }^{1}$
}

Received: 20 August 2019 / Revised: 6 October 2019 / Accepted: 7 October 2019 / Published online: 19 December 2019

(c) The Author(s), under exclusive licence to United States \& Canadian Academy of Pathology 2019

\begin{abstract}
Human papillomavirus (HPV) is an oncogenic virus associated with the development of several human cancers. Primary vaginal, vulvar, and anal adenocarcinomas are rare and, to date, have rarely been shown to be associated with HPV infection. We report a series of nine HPV-related adenocarcinomas of the lower anogenital tract distal to the cervix. The tumors involved the vagina (4), anorectum (3), and vulva (2). Two of the three anorectal cases involved men. Patients presented with a vulvar or vaginal mass/nodule, painless rectal bleeding, or during screening colonoscopy. Lesions ranged in size from 3.2 to $8.4 \mathrm{~cm}$. The most salient morphologic characteristic was the presence of papillary or villiform/villoglandular architecture in all cases. Tumors displayed features similar to those of usual type high-risk HPV-related endocervical adenocarcinoma, namely, mucinous or mucin-poor (pseudoendometrioid) features or a hybrid of these, with columnar cells with crowded, cigar-shaped to ovoid irregular nuclei. Mitoses (mostly apical) and apoptotic bodies were easily identified. Adenosis was present in two vaginal cases. One anal tumor featured abundant intracytoplasmic mucin that was multivacuolated in some areas imparting a "clear cell"-like appearance. All tumors were diffusely and strongly positive for p16. Seven of seven tested cases were positive for high-risk HPV by in situ hybridization or polymerase chain reaction. Follow-up information, available in five patients, revealed two local recurrences but no tumor related deaths or distant metastases. We report the first well-documented series of HPV-associated primary adenocarcinomas of the vagina, vulva, and anorectum and broaden the spectrum of HPV-related neoplasia involving the lower anogenital tract in both women and men.
\end{abstract}

These authors share senior authorship: Teri A. Longacre, Brigitte M. Ronnett

Lysandra Voltaggio

lvoltag1@jhmi.edu

1 Department of Pathology, Johns Hopkins University School of Medicine, Baltimore, MD, USA

2 Department of Pathology, Belfast Health and Social Care Trust, Belfast, UK

3 Department of Pathology, MedStar Franklin Square Medical Center, Baltimore, MD, USA

4 Department of Pathology, Stanford University, Stanford, CA, USA

\section{Introduction}

High-risk human papillomaviruses (HPV) are oncogenic viruses associated with the development of several human cancers. Based on Center for Disease Control data, about 42,700 new cases of high-risk HPV-associated cancers occurred in the United States each year from 2011 to 2015 [1]. The most common site of high-risk HPV-related cancer in women is the cervix, while oropharyngeal carcinoma is most common in men. Carcinoma of the vagina, vulva, penis, and anus occur less frequently. While infection mostly results in development of squamous cell carcinoma, high-risk HPV less commonly drives the development of glandular lesions, including a large majority of endocervical adenocarcinomas.

Primary vaginal, vulvar, and anal adenocarcinomas are rare and, to date, documentation of examples related to high-risk HPV is scant [2-8]. Vaginal adenocarcinoma was, in the past, most commonly associated with adenosis related 
to diethylstilbestrol exposure in utero (prescribed during 1938-1971 to prevent miscarriages and other pregnancy issues). Such tumors are mostly clear cell carcinomas unassociated with high-risk HPV, and cases related to this medication are essentially no longer encountered by practicing pathologists. Primary vaginal clear cell carcinomas may occur sporadically and are not high-risk HPV-related. Endometrioid-type and intestinal-type adenocarcinomas of the vagina unrelated to high-risk HPV have also been reported $[9,10]$. In addition, primary vaginal gastric-type adenocarcinomas unrelated to high-risk HPV, similar to those occurring more commonly in the cervix, have recently been reported [11-13]. Primary vulvar adenocarcinomas are extremely rare with the existing literature limited to case reports, with one case reportedly associated with high-risk HPV [4, 14-16]. Primary anal canal adenocarcinomas are exceptional, with less than a handful of articles published to date $[17,18]$. The potential association of high-risk HPV has not been widely studied in anal adenocarcinoma, although Meriden and Montgomery [18] reported four anal duct carcinomas that were negative for high-risk HPV by in situ hybridization.

In this study, we report nine cases of primary vaginal, vulvar, and anorectal high-risk HPV-associated adenocarcinomas. We describe the morphologic and immunohistochemical features and stress the similarity to those of high-risk HPV-related endocervical adenocarcinoma.

\section{Materials and methods}

Nine cases were identified from the gastrointestinal and gynecologic pathology files of three institutions (Johns Hopkins Hospital [two cases], Stanford [five cases], and Belfast [two cases]). Eight were consults and one was an inhouse case. All available pathology reports and slides were reviewed. Clinical data and follow-up information were obtained from the charts, contributing pathologists, or clinicians. Various immunohistochemical markers were performed at the different institutions during the reporting of the cases. The immunohistochemistry was performed in multiple institutions using different antibodies and methods. For p16, block-type immunoreactivity was defined as diffuse nuclear and cytoplasmic staining of essentially all tumor cells, while lesser staining than this was considered negative (non-block-type). For the other markers, staining was classified as negative, focal $(<50 \%$ cells staining) or diffuse ( $>50 \%$ cells staining).

HPV studies were performed in seven cases using either in situ hybridization or polymerase chain reaction. For the in situ hybridization, older cases were assessed for HPV by DNA in situ hybridization, whereas more recent cases were assessed by RNA in situ hybridization. HPV DNA in situ hybridization was using biotin-labeled HPV probe solutions (Dako Corporation, Carpinteria, CA) applied to individual sections. These included a wide spectrum probe (cocktail of HPV 6, 11, 16, 18, 31, 33, 35, 45, 51, and 52) and separate type-specific probes for HPV 16 and HPV 18. Detection of hybridized probe was performed by tyramide catalyzed signal amplification using the Dako Genpoint Kit (Dako). Chromogenic detection was performed with 3,3'-diaminobenzidine/H2O2. Controls included tissue sections positive for HPV wide spectrum, the HeLa cell line for HPV 18 and the SiHa cell line for HPV 16. Biotin-labeled plasmid probes served as a negative control in each case. Cases with a discrete punctate reaction product specifically in tumor cell nuclei were interpreted as positive. The chromogenic ISH RNAscope ${ }^{\circledR}$ assay was performed on a Leica Bond Autostainer (Leica Biosystems, Wetzlar, Germany) according to the manufacturer's protocol. In brief, $4 \mu \mathrm{m}$ tissue sections were prepared using standard procedures. Slides were deparaffinized, rehydrated, and pretreated with RNAscope ${ }^{\circledR}$ Target Retrieval at $95^{\circ} \mathrm{C}$ for $15 \mathrm{~min}$ followed by treatment with RNAscope ${ }^{\circledR}$ Enzyme for $15 \mathrm{~min}$ (Advanced Cell Diagnostics, Newark, CA). Slides were hybridized for 120 min followed by amplification of signal and detection using the RNAscope ${ }^{\circledR} 2.5$ HD Brown Kit (Advanced Cell Diagnostics, Newark, CA) and then hematoxylin counterstained and mounted. The probe used for detection was a commercially available prediluted RNAscope $^{\circledR}$ 2.5 LS Probe-HPV-HR18 (Catalog number: 312598, Advanced Cell Diagnostics, Newark, CA) targeting HPV strains $16,18,26,31,33,35,39,45,51,52,53$, $56,58,59,66,68,73$, and 82, E6/E7. Human tissue with confirmed HR-HPV infection was used as a positive control and a PPIB control probe was used to test RNA quality. Cases with discrete punctate reaction product specifically in tumor cells were interpreted as positive.

Polymerase chain reaction analysis was performed on some cases either to investigate a suspected failure to detect HPV by in situ hybridization or because the reporting laboratory used this as the HPV detection method. For case 1, the SPF-10 LiPA HPV typing method was used [19]. To extract the DNA, paraffin was solubilized with octane by shaking at room temperature for 15-30 min. The tissue was pelleted by centrifugation, and washed with $100 \%$ ethanol. After removing the ethanol after centrifugation, the tissue was dried with the addition of $10 \mu \mathrm{L}$ acetone in a $55^{\circ} \mathrm{C}$ heating block. Dried tissues were resuspended in a $1 \times$ digestion buffer of $200 \mu \mathrm{g} / \mathrm{mL}$ proteinase $\mathrm{K}$ and $0.1 \%$ laureth- 12 and incubated at $55{ }^{\circ} \mathrm{C}$ until the tissue was fully digested. Protease was heatinactivated by incubation at $95{ }^{\circ} \mathrm{C}$ for $10 \mathrm{~min}$. The SPF- 10 LiPA HPV typing was performed according the manufacturer's instructions (DDL Diagnostics Laboratory, The Netherlands). The SPF-10-line probe assay is a 
broad-spectrum short-fragment polymerase chain reaction (SPF-10 PCR) that recognizes most genital tract HPV types by amplifying a conserved $65 \mathrm{bp}$ fragment of the L1 region of the HPV genome, the smallest amplicon of any HPV DNA typing system available [19, 20]. For case 3, polymerase chain reaction amplification was performed using primers and a fluorescein-labeled hydrolysis probe specific for the HPV 16 E6 gene, as well as primers and a Cal Fluor Orange-560-labeled probe specific for the HPV 18 E6 gene. For cases 7 and 8, linear array HPV genotyping (Roche Molecular Diagnostics, Pleasanton, California, USA) was carried out. The Roche linear array HPV genotyping test involves polymerase chain reaction amplification of target DNA followed by hybridization for the detection of $37 \mathrm{HPV}$ types, 18 high-risk types $(16,18$, $26,31,33,35,39,45,51,52,53,56,58,59,66,68,73$, $82)$, and 19 low-risk types $(6,11,40,42,54,55,61,62$, 64, 67, 69, 70, 71, 72, 81, 83, 84, IS39, CP108).

The study was approved by our Institutional Review Board.

\section{Results}

\section{Clinical features}

Clinicopathologic features of the cases are presented in Table 1. This series included nine cases (seven excisions/ resections, two biopsies, and one procedure not specified) from seven women and two men. In one case (case 1) both the primary tumor and a recurrence were available for the study. The average patient age was 50.2 years (range, 38-69). Seven patients were Caucasian, one African American, and one Indian. Where information was available, presentation included the presence of a vulvar (one labial, one unspecified) or vaginal mass/nodule (3), painless rectal bleeding (1), or the lesion was identified during screening colonoscopy (1). Imaging studies were available for four patients. Patient 2 had an adrenal lesion on positron emission tomography concerning for metastatic disease; adrenalectomy showed an adrenocortical adenoma. Patient 5 showed no evidence of metastatic disease (uncertain imaging modality). Patients 3 and 7 showed no additional lesions on magnetic resonance imaging and positron emission tomography. Two women underwent loop excision of the cervix (patient 1: negative; patient 7: high-grade squamous intraepithelial lesion [cervical intraepithelial neoplasia grade 2]). Patient 2, a woman with an anorectal tumor, had no information regarding the status of the cervix but the clinician felt that the lesion was limited to the anorectum and histologically the pattern of growth was consistent with a primary anorectal lesion.

\section{Pathologic features}

A gross description was available for five cases, and the tumors ranged in size from 3.2 to $8.4 \mathrm{~cm}$. Various descriptions of the cut surface included "granular", "red", "polypoid", "lobular", "rubbery", "pedunculated", and "soft to firm".

Histologically, a papillary or villiform/villoglandular architecture was present in all nine cases and typically predominated; a glandular (nonpapillary) architecture was also focally present in all cases (Fig. 1a-c). Focal cribiforming was seen in one case each from the vulva and vagina (Fig. 2a). Deep stromal invasion and/or lymphovascular invasion were present in three vaginal and vulvar cases (cases 1,6, and 8). The anal lesions were polypoid and predominantly exophytic, replaced most of the squamous mucosa, and merged with the adjacent rectal epithelium (Fig. 2b). Of the anal cases, only one (case 3) featured focal superficial submucosal invasion. Three cases, one from the vulva, one from the vagina, and one from the anorectum, showed associated high-grade squamous intraepithelial lesion. Lymphoid aggregates were prominent immediately underneath the tumor in one anorectal case (Fig. 1b). All tumors showed characteristic morphologic features of high-risk HPV-related, usual type endocervical adenocarcinoma with papillary, or villiform/villoglandular fronds lined by columnar cells with mucinous or mucinpoor features with crowded, cigar-shaped to ovoid irregular nuclei. Mucin depletion led to an appearance that simulated endometrioid differentiation, but these tumors are not endometrioid. Some observers use the term "pseudoendometrioid" to refer to this mucin-poor pattern. Chromatin quality varied from delicate with pinpoint nucleoli to focally vesicular to hyperchromatic without visible nucleoli (Fig. 2c, d). Mitoses (mostly apical) and apoptotic bodies were easily identified (Fig. 2d). Nuclear to cytoplasmic ratio, as well as intracytoplasmic mucin content, were variable between and even within tumors. One anal tumor featured abundant intracytoplasmic mucin that was in the form of goblet cells in some areas and multivacuolated in others imparting a "clear cell"-like appearance (Fig. 2e, f). In lesions from the vulva and vagina, mucin was generally less prominent with focal goblet cells in some cases (Fig. 3a), while some areas were totally devoid of intracytoplasmic mucin (Fig. 3b, c). Adenosis, comprising benign mucinous glands, was present in two vaginal cases (1 and 8 ); this was seen in both the original tumor and the recurrence in patient 1 (Fig. 4a, b).

\section{Immunohistochemical findings and HPV results}

The immunohistochemical and HPV results are summarized in Table 2. All tumors were diffusely and strongly positive 


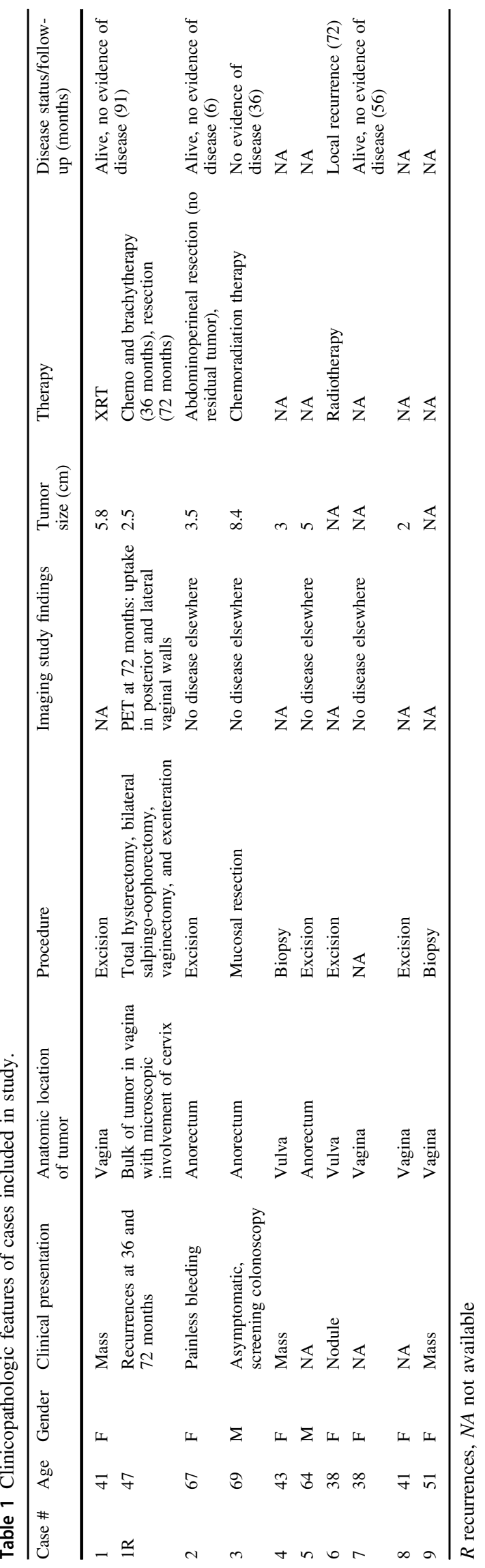

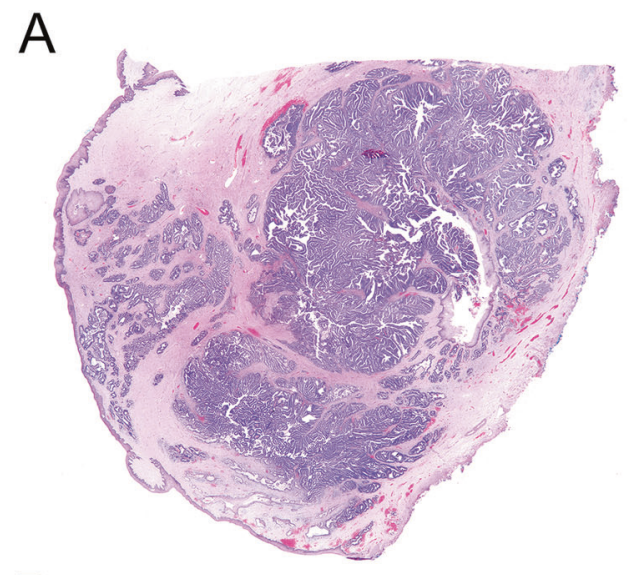
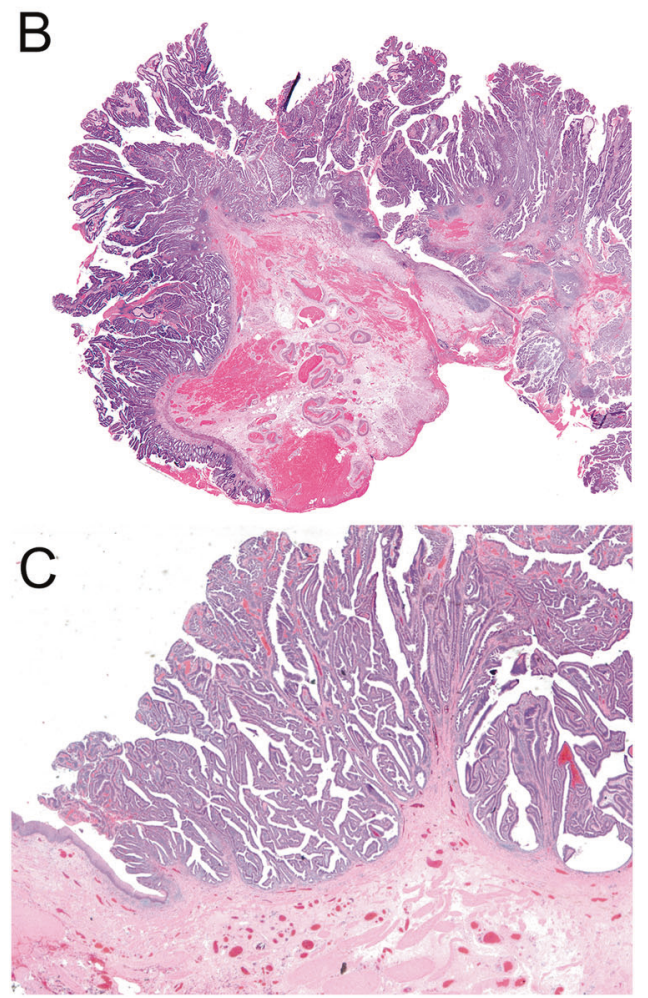

Fig. 1 A papillary or villiform/villoglandular architecture was present in all cases. Examples from the vagina (a) and anorectum $(\mathbf{b}, \mathbf{c})$.

for p16 (block-type immunoreactivity) (Fig. 5a). CK7 was performed in seven cases, all of which were diffusely immunoreactive (Fig. 5b). CDX2 was at least focally positive in all six cases tested (two anorectal, one vulvar, and three vaginal). PAX8 was performed in four cases and was positive in one vaginal tumor and negative in two anorectal and one vulvar tumor. CK20 was focally positive in one tumor each from the vulva and vagina, while two anorectal and two vaginal tumors were negative. ER and PR were negative $(0 / 5)$ as was WT1 (0/2) in all cases tested. Additional negative stains included vimentin ( $0 / 2$ vaginal), synaptophysin (0/2 vaginal), Napsin A (0/1 vaginal), and 
Fig. 2 Cribiforming was seen in two cases (a). The anal lesions (right side of the mucosa) merged with the adjacent rectal epithelium (b). Tumors featured columnar cells with mucinous (c) or mucinpoor (pseudoendometrioid) features; apical mitoses and apoptotic bodies were easily identified (d). Anal tumor with abundant intracytoplasmic mucin that was in the form of goblet cells (e) in some areas and multivacuolated in others (f) imparting a clear cell appearance.
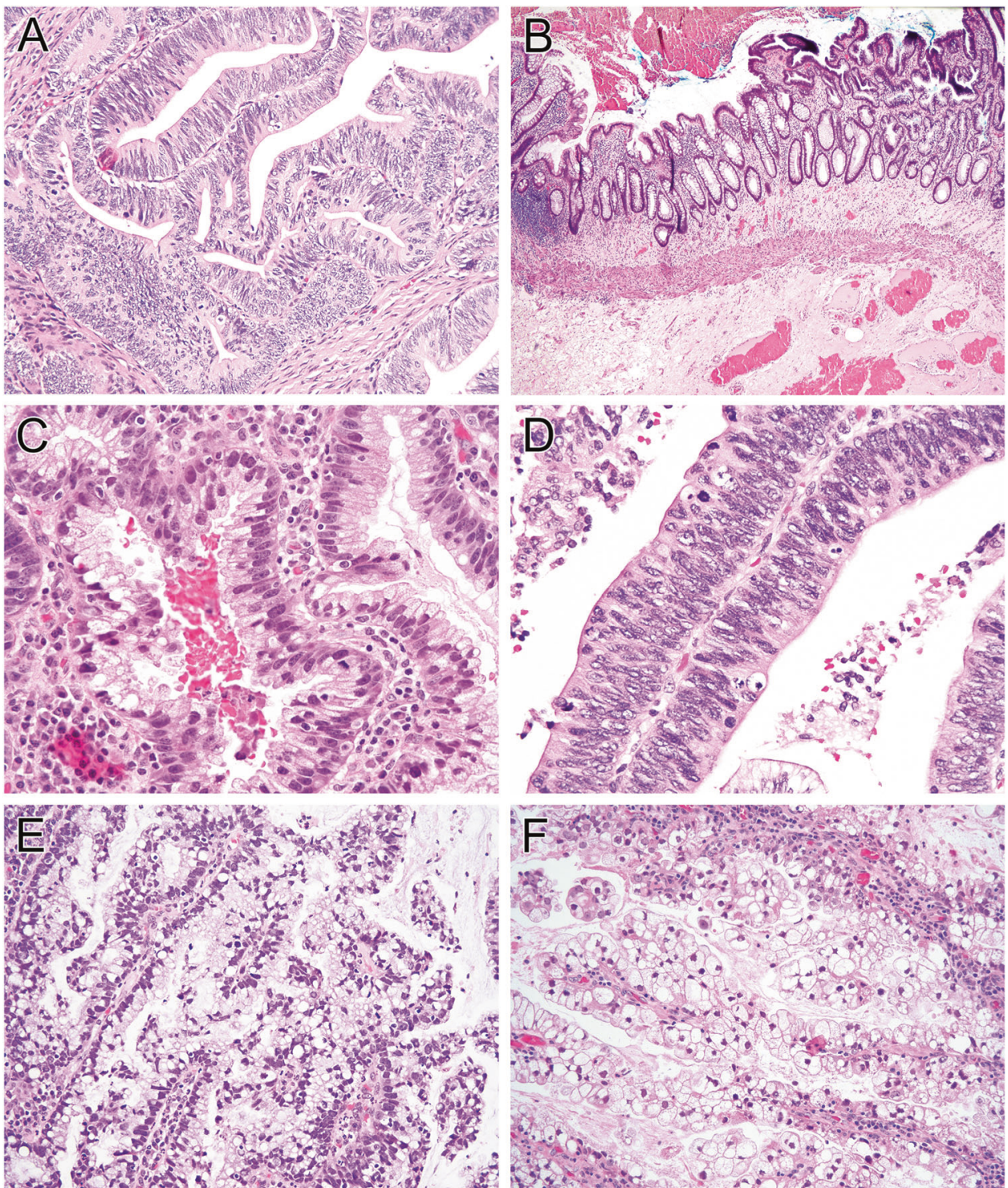

TTF1 (0/1 vulvar). P53 showed wild-type immunoreactivity in one vaginal tumor tested. Immunohistochemical stains for mismatch repair proteins (MLH1, PMS2, MSH2, and MSH6), performed in one anal case, showed intact nuclear expression with all four markers.

For all seven cases tested, high-risk HPV was detected in the tumors (two by ISH, five by PCR). Of three cases tested with high-risk HPV in situ hybridization, two had detectable HPV (Fig. 5c). HPV 16 was detected in one of these (vulvar). Case 2 (anorectal) was tested with a high risk-HPV cocktail; thus, the exact HPV type could not be determined. High-risk HPV was not detected by in situ hybridization in case 1 (vagina), but HPV 31 was found by polymerase chain reaction, underscoring the imperfect sensitivity of in situ hybridization assay. Three cases tested only by polymerase chain reaction had high-risk HPV detected (one with HPV 16 and two with HPV 18).

\section{Patient follow-up}

Follow-up information was available for five patients (5-91 months, mean 51). Patient 1 experienced parametrial and vaginal recurrences 3 and 6 years, respectively, after the initial vaginal excision and chemoradiation. The patient eventually underwent a total hysterectomy, bilateral salpingo-oophorectomy, vaginectomy, and distal colectomy, where the bulk of the tumor was located in the upper third of the vagina with focal involvement of the cervix and perirectal soft tissue. The tumor was deemed clinically a primary vaginal adenocarcinoma (no tumor was present in the original cervical loop biopsy); the patient was alive at the time of last follow-up. Patient 2 underwent abdominoperineal resection following initial polypectomy, which showed no residual tumor; she was alive 5 months after the initial diagnosis. Patient 3 was alive without disease 


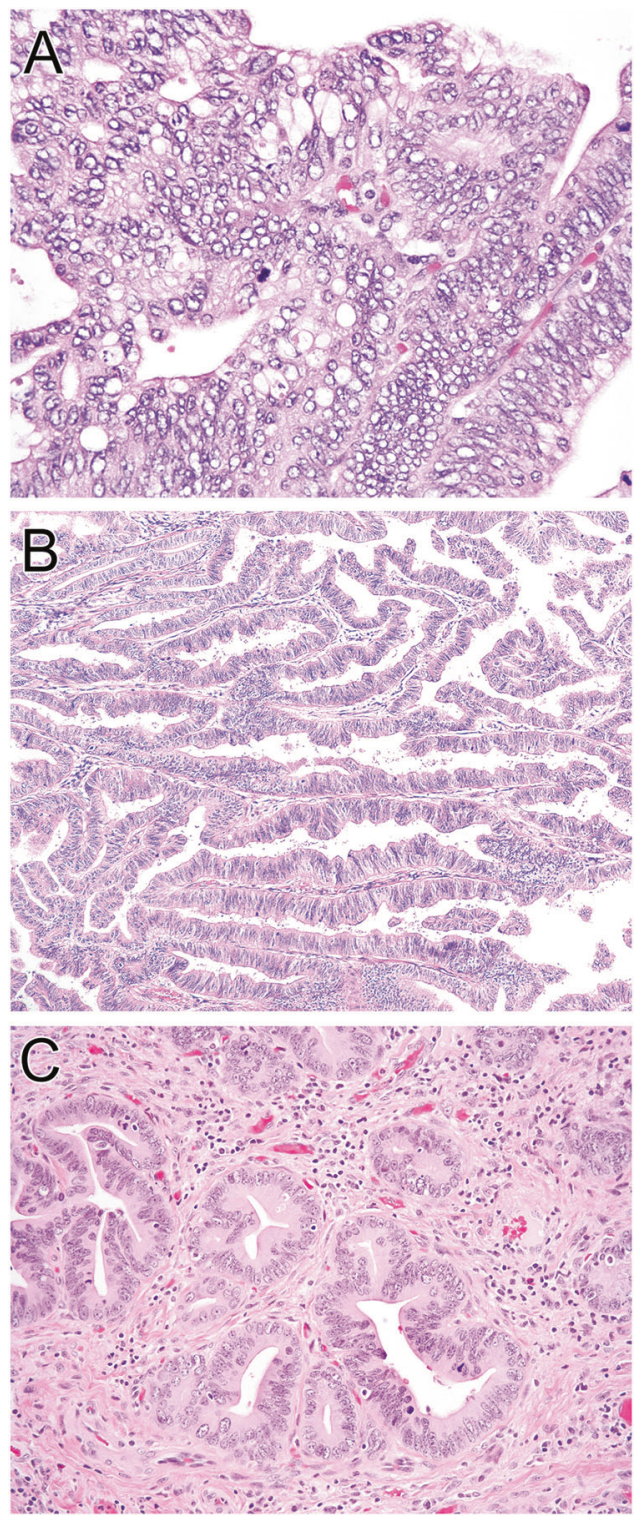

Fig. 3 Mucin was less prominent in tumors from the vulva and vagina with focal goblet cells in some cases (a), while some areas were totally devoid of intracytoplasmic mucin (b, c).
36 months after the initial diagnosis. Patient 6 experienced local recurrence 7 years after the initial diagnosis. Patient 7 was alive and free of disease 56 months after diagnosis.

\section{Discussion}

Primary anal, vaginal, and vulvar glandular lesions are rare and, until recently, association with high-risk HPV has not been well-documented/established. In this report, we describe a novel group of high-risk HPV-associated adenocarcinomas of the lower anogenital tract in both men and women, with those in women being distal to the cervix; the morphological features of the tumors in both men and women closely resembled high-risk HPV-associated endocervical adenocarcinomas.

Primary vaginal adenocarcinomas are, as discussed, rare but have been documented [10, 12, 21, 22]. The current 2014 World Health Organization classification of vaginal carcinomas includes categories of endometrioid carcinoma, clear cell carcinoma, mesonephric carcinoma, and mucinous carcinoma; the latter are defined as resembling either endocervical or intestinal-type carcinoma [23], similar to those found in the cervix. However, vaginal tumors resembling endocervical-type adenocarcinomas are not well described and we are unable to find a single report in the literature of an HPV-associated primary vaginal adenocarcinoma morphologically resembling an HPV-related endocervical adenocarcinoma of usual type. The largest reported series of primary vaginal glandular lesions (14 cases) unassociated with adenosis, reported by Staats et al. [10], described intestinal-type glandular lesions composed of cells with abundant, mucin-filled cytoplasm, and numerous goblet cells. These lesions displayed intestinaltype features, including predominantly diffuse CK20 and CDX2 immunoreactivity and variable (negative to focal to diffuse) CK7 expression. p16 was negative in the two cases they tested and HPV in situ hybridization or polymerase
Fig. 4 Adenosis was seen in the original tumor (a) and the recurrence $(\mathbf{b})$ of patient 1 .
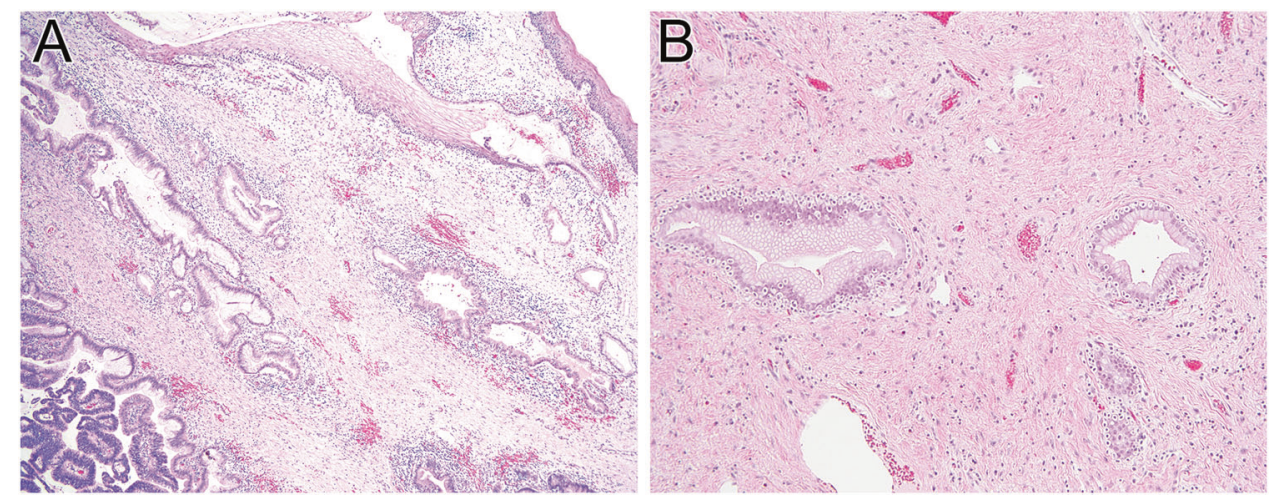
Table 2 Ancillary immunohistochemical and HPV testing results.

\begin{tabular}{|c|c|c|c|c|c|c|c|c|c|c|}
\hline Case \# & Anatomic site & p16 & HPV ISH & HPV PCR & CK7 & CK20 & $\mathrm{CDX} 2$ & Pax8 & ER & PR \\
\hline 1 & Vagina & + (Diffuse) & - & + (HPV 31) & NP & NP & NP & NP & - & - \\
\hline $1 \mathrm{R}$ & Vagina & NP & NP & NP & NP & NP & NP & NP & NP & NP \\
\hline 2 & Anorectum & + (Diffuse) & + & NP & + & - & $+($ Focal $)$ & - & - & - \\
\hline 3 & Anorectum & + (Diffuse) & NP & + (HPV 18) & + & - & $+($ Focal $)$ & - & NP & NP \\
\hline 4 & Vulva & $+($ Diffuse $)$ & + (HPV 16) & NP & + & $+($ Focal $)$ & $+($ Focal $)$ & - & - & NP \\
\hline 5 & Anorectum & + (Diffuse) & NP & NP & NP & NP & NP & NP & NP & NP \\
\hline 6 & Vulva & + (Diffuse) & NP & NP & + & NP & NP & NP & NP & NP \\
\hline 7 & Vagina & + (Diffuse) & NP & + (HPV 16) & + & - & $+($ Focal $)$ & + & - & NP \\
\hline 8 & Vagina & + (Diffuse) & NP & + (HPV 18) & + & $+($ Focal $)$ & + & NP & - & - \\
\hline 9 & Vagina & + (Diffuse) & NP & $+($ Cocktail $)$ & + & - & $+($ Focal $)$ & NP & NP & NP \\
\hline
\end{tabular}

$H P V$ human papillomavirus, $I S H$ in situ hybridization, $P C R$ polymerase chain reaction, $N P$ not performed

chain reaction was not performed [10]. Additional case reports of primary vaginal adenocarcinoma either feature dissimilar morphology to the adenocarcinomas we report and/or HPV is not detected or information regarding HPV status is lacking [12, 21, 22]. In contrast, the cases we herein describe display distinctive morphologic and immunohistochemical features as well as an association with high-risk HPV.

Recently, in an article coauthored by one of us (WGM), Wong et al. [13], described five cases of primary vaginal gastric-type adenocarcinoma, identical to those neoplasms which are well characterized within the cervix. These cases were all associated with adenosis, which exhibited gastric differentiation and featured abundant clear and/or eosinophilic, focally foamy cytoplasm; basaloid and sarcomatoid components were present in one case. p16 was negative (non-block-type immunoreactivity) in all the cases, and these represent non-HPV-associated neoplasms which, as discussed, are identical to gastric-type adenocarcinoma of the cervix, which is typically negative or focally positive for p16 and unrelated to high-risk HPV [24-26]. In this paper, the authors also reported cases of vaginal adenosis unassociated with tumor and exhibiting gastric differentiation. They speculated on two pathways of primary vaginal adenocarcinoma arising in adenosis, namely clear cell carcinomas arising in tuboendometrial-type adenosis and gastrictype adenocarcinomas arising in adenosis exhibiting gastric differentiation. Interestingly, benign glands, in keeping with adenosis, were present in two of our primary vaginal adenocarcinomas suggesting that a third type of primary vaginal adenocarcinoma (HPV-related) may potentially arise in adenosis.

It is worth mentioning that a large majority of vaginal adenocarcinomas will be metastatic, most commonly from other sites in the female genital tract, especially the endometrium and cervix, and also from the colorectum. In diagnosing a primary vaginal high-risk HPV-associated adenocarcinoma one should not solely rely on p16 staining since some other female genital tract adenocarcinomas, especially high-grade serous carcinomas, can exhibit diffuse immunoreactivity secondary to non-HPV-related factors [27, 28]. In addition, one of us (WGM) has observed diffuse immunoreactivity with p16 in primary colorectal adenocarcinoma [29]. It is also emphasized that p16 staining should be strong and diffuse (block-type) in order to support a diagnosis of a high-risk HPV-associated neoplasm (with only rare exceptions); ideally, in situ hybridization or polymerase chain reaction is desirable to confirm this.

Primary vulvar adenocarcinoma is extremely rare. Reported cases describe mucinous [30], signet ring cell [31], intestinal [16, 32, 33], and clear cell [15] morphology. A single case report described villoglandular morphology, similar to our cases, but differed in demonstrating diffuse CK20 immunoreactivity and lack of CK7 expression. Results of high risk-HPV testing were not provided [34]. An association with high-risk HPV has been documented in one case of vestibular adenocarcinoma in situ, thought to have arisen from an adjacent minor vestibular gland [4].

While an association with anal squamous cell carcinoma is well established, the relationship between high-risk HPV and anal adenocarcinoma has not been widely explored, likely owing to the rarity of this neoplasm. Koulos et al. [6] detected HPV 18 by polymerase chain reaction in 2 of 6 anal adenocarcinomas and in none of seven adenocarcinomas of the colon and rectum. Recent data supports high-risk HPV-dependent and -independent pathways for the development of anal glandular neoplasia. Herfs et al. demonstrated high-risk HPV by PCR and ISH in 11 of 26 anal gland/transitional zone-type adenocarcinomas but detailed histologic descriptions of the tumors were not provided [7]. On the other hand, high-risk HPV DNA was absent in all 48 colorectal-type anal adenocarcinomas tested [7]. In a similar vein, recent work by Shamir et al. demonstrated high-riskHPV-related Rb and TP53 inactivation in 7/25 colorectal 


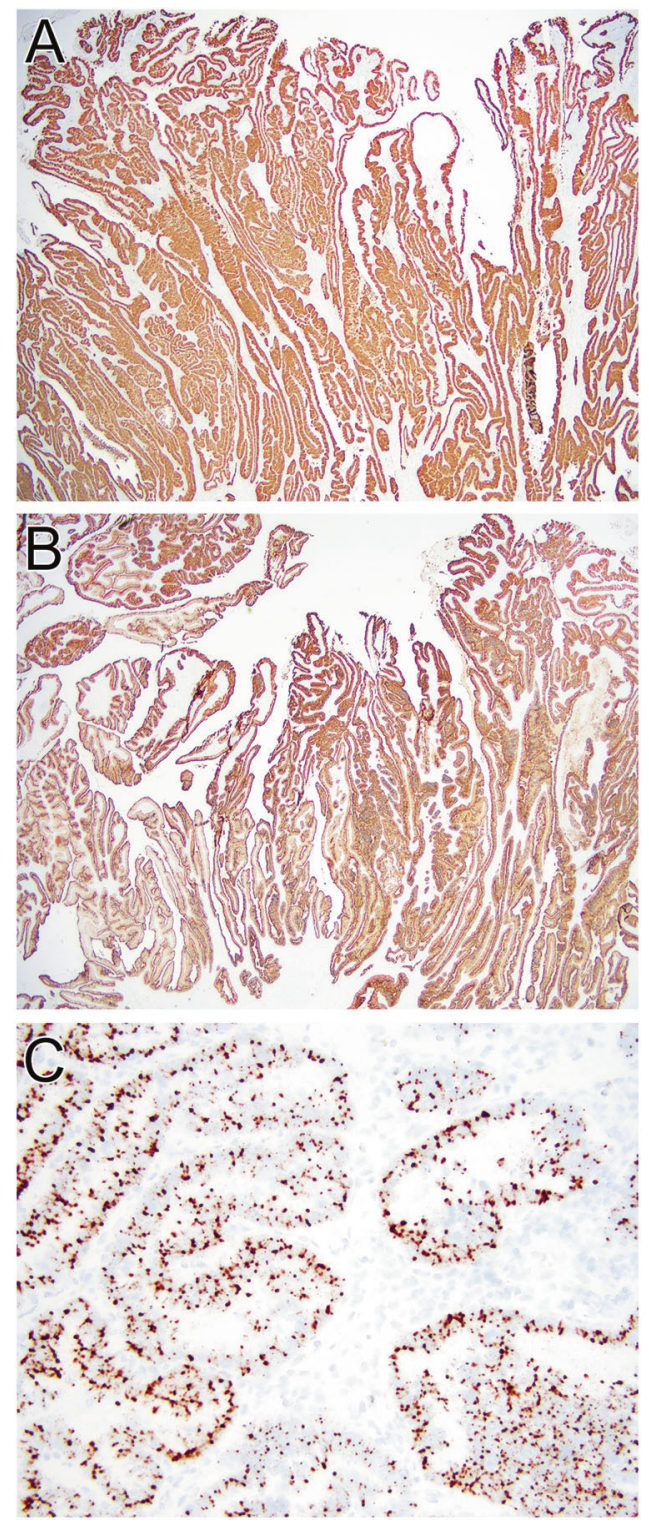

Fig. 5 All tumors were diffusely and strongly positive for p16 (a). CK7 was diffusely immunoreactive in all seven cases tested (b). HRHPV RNA in situ hybridization was detected in this case from the anorectum (c).

and anal neuroendocrine carcinomas, all localized to the rectum and anus [8].

One could argue that an HPV association cannot be definitely proven for cases 5 and 6 , where HPV studies were not performed (material not available for testing) and that p16 immunoreactivity may reflect epigenetic or genetic alterations in the $R b$ gene or another type of dysregulation of the $\mathrm{Rb}$ pathway which, as already discussed, can occur in other tumor types. However, given the striking morphologic similarity to primary endocervical HPV-associated adenocarcinomas, similar to our other high-risk HPV-proven cases described herein, we have included these cases in our study.
While a subset of our patients developed local recurrences, the clinical course was indolent in most patients for whom follow-up was available. However, this series is too small to draw solid conclusions regarding tumor behavior and larger multi-institutional studies are needed to better characterize these rare neoplasms, determine their behavior, and to delineate effective treatment strategies.

In summary, we report the first series of high-risk-HPVrelated adenocarcinomas of the lower anogenital tract (vagina, vulva, and anorectum). These are rare but morphologically distinctive tumors that exhibit papillary or villiform/villoglandular morphology and cytologic features similar to those of usual type, high-risk HPV-related endocervical adenocarcinomas.

Acknowledgements We are indebted to Ms Kara Lombardo and $\mathrm{Mr}$ Hiro Nonogaki for their assistance with methodology and to Norm Barker, MA, MS, RBP, FBCA, FRPS for his assistance with the photomicrographs.

\section{Compliance with ethical standards}

Conflict of interest The authors declare that they have no conflict of interest.

Publisher's note Springer Nature remains neutral with regard to jurisdictional claims in published maps and institutional affiliations.

\section{References}

1. Centers for Disease Control and Prevention. Cancers associated with human papilloma virus, United States-2011-2015. USCS data brief, no. 4. Atlanta, GA: Centers for Disease Control and Prevention; 2018.

2. Ikenberg $H$, Runge $M$, Göppinger A, Pfleiderer A. Human papillomavirus DNA in invasive carcinoma of the vagina. Obstet Gynecol. 1990;76:432-8.

3. Waggoner SE, Anderson SM, Van Eyck S, Fuller J, Luce MC, Herbst AL. Human papillomavirus detection and p53 expression in clear-cell adenocarcinoma of the vagina and cervix. Obstet Gynecol. 1994;84:404-8.

4. Talia KL, Otton G, Garland S, Phillips S, Scurry J. Human papillomavirus-associated adenocarcinoma in-situ of the vestibule. J Low Genit Trac Dis. 2017;21:e45-7.

5. Paczos TA, Ackers S, Odunsi K, Lele S, Mhawech-Fauceglia P. Primary vaginal adenocarcinoma arising in vaginal adenosis after $\mathrm{CO} 2$ laser vaporization and 5-fluorouracil therapy. Int J Gynecol Pathol. 2010;29:193-6.

6. Koulos J, Symmans F, Chumas J, Nuovo G. Human papillomavirus detection in adenocarcinoma of the anus. Mod Pathol. 1991;4:58-61.

7. Herfs M, Roncarati P, Koopmansch B, Peulen O, Bruyere D, Lebeau A, et al. A dualistic model of primary anal canal adenocarcinoma with distinct cellular origins, etiologies, inflammatory microenvironments and mutational signatures: implications for personalised medicine. Br J Cancer. 2018;118:1302-12.

8. Shamir ER, Devine WP, Pekmezci M, Umetsu SE, Krings G, Federman S, et al. Identification of high-risk human papillomavirus and $\mathrm{Rb} / \mathrm{E} 2 \mathrm{~F}$ pathway genomic alterations in mutually 
exclusive subsets of colorectal neuroendocrine carcinoma. Mod Pathol. 2019;32:290-305.

9. Staats PN, Clement PB, Young RH. Primary endometrioid adenocarcinoma of the vagina: a clinicopathologic study of 18 cases. Am J Surg Pathol. 2007;31:1490-501.

10. Staats PN, McCluggage WG, Clement PB, Young RH. Primary intestinal-type glandular lesions of the vagina: clinical, pathologic, and immunohistochemical features of 14 cases ranging from benign polyp to adenoma to adenocarcinoma. Am J Surg Pathol. 2014;38:593-603.

11. Carleton C, Hoang L, Sah S, Kiyokawa T, Karamurzin YS, Talia $\mathrm{KL}$, et al. A detailed immunohistochemical analysis of a large series of cervical and vaginal gastric-type adenocarcinomas. Am J Surg Pathol. 2016;40:636-44.

12. Talia KL, Scurry J, Manolitsas T, McCluggage WG. Primary vaginal mucinous adenocarcinoma of gastric type arising in adenosis: a report of 2 cases, 1 associated with uterus didelphys. Int $\mathbf{J}$ Gynecol Pathol. 2012;31:184-91.

13. Wong RW, Moore M, Talia KL, Ganesan R, McCluggage WG. Primary vaginal gastric-type adenocarcinoma and vaginal adenosis exhibiting gastric differentiation: report of a series with detailed immunohistochemical analysis. Am J Surg Pathol. 2018;42:958-70.

14. Cormio G, Carriero C, Loizzi V, Gissi F, Leone L, Putignano G, et al. "Intestinal-type" mucinous adenocarcinoma of the vulva: a report of two cases. Eur J Gynaecol Oncol. 2012;33:433-5.

15. Kojima N, Yoshida H, Uehara T, Ushigusa T, Asami Y, Shiraishi KT, et al. Primary clear cell adenocarcinoma of the vulva: a case study with mutation analysis and literature review. Int J Surg Pathol. 2019;27:792-7. 1066896919848823.

16. Kurita T, Matuura Y, Hisaoka M, Hachisuga T. Adenocarcinoma of intestinal type of the vulva. Int Cancer Conf J. 2019;8:89-93.

17. Lisovsky M, Patel K, Cymes K, Chase D, Bhuiya T, Morgenstern N. Immunophenotypic characterization of anal gland carcinoma: loss of p63 and cytokeratin 5/6. Arch Pathol Lab Med. 2007;131:1304-11.

18. Meriden Z, Montgomery EA. Anal duct carcinoma: a report of 5 cases. Hum Pathol. 2012;43:216-20.

19. Kleter B, van Doorn LJ, ter Schegget J, Schrauwen L, van Krimpen K, Burger M, et al. Novel short-fragment PCR assay for highly sensitive broad-spectrum detection of anogenital human papillomaviruses. Am J Pathol. 1998;153:1731-9.

20. Kleter B, van Doorn LJ, Schrauwen L, Molijn A, Sastrowijoto S, ter Schegget J, et al. Development and clinical evaluation of a highly sensitive PCR-reverse hybridization line probe assay for detection and identification of anogenital human papillomavirus. $\mathbf{J}$ Clin Microbiol. 1999;37:2508-17.

21. Saitoh M, Hayasaka T, Ohmichi M, Kurachi H, Motoyama T. Primary mucinous adenocarcinoma of the vagina: possibility of differentiating from metastatic adenocarcinomas. Pathol Int. 2005;55:372-5.

22. McCluggage WG, Price JH, Dobbs SP. Primary adenocarcinoma of the vagina arising in endocervicosis. Int $\mathrm{J}$ Gynecol Pathol. 2001;20:399-402.

23. urman RJ, Carcangiu ML, Herrington CS, Young RH WHO classification of tumors of female reproductive organs. Lyon: IARC; 2014

24. Kojima A, Mikami Y, Sudo T, Yamaguchi S, Kusanagi Y, Ito M, et al. Gastric morphology and immunophenotype predict poor outcome in mucinous adenocarcinoma of the uterine cervix. Am J Surg Pathol. 2007;31:664-72.

25. Kusanagi Y, Kojima A, Mikami Y, Kiyokawa T, Sudo T, Yamaguchi S, et al. Absence of high-risk human papillomavirus (HPV) detection in endocervical adenocarcinoma with gastric morphology and phenotype. Am J Pathol. 2010;177:2169-75.

26. Wada T, Ohishi Y, Kaku T, Aman M, Imamura H, Yasutake N, et al. Endocervical adenocarcinoma with morphologic features of both usual and gastric types: clinicopathologic and immunohistochemical analyses and high-risk HPV detection by in-situ hybridization. Am J Surg Pathol. 2017;41:696-705.

27. Chiesa-Vottero AG, Malpica A, Deavers MT, Broaddus R, Nuovo GJ, Silva EG. Immunohistochemical overexpression of p16 and p53 in uterine serous carcinoma and ovarian high-grade serous carcinoma. Int J Gynecol Pathol. 2007;26:328-33.

28. Yemelyanova A, Ji H, Shih IeM, Wang TL, Wu LS, Ronnett BM. Utility of p16 expression for distinction of uterine serous carcinomas from endometrial endometrioid and endocervical adenocarcinomas: immunohistochemical analysis of 201 cases. Am J Surg Pathol. 2009;33:1504-14.

29. Lam AK, Ong K, Giv MJ, Ho YH. p16 expression in colorectal adenocarcinoma: marker of aggressiveness and morphological types. Pathology. 2008;40:580-5.

30. Sui Y, Zou J, Batchu N, Lv S, Sun C, DU J, et al. Primary mucinous adenocarcinoma of the vulva: a case report and review of the literature. Mol Clin Oncol. 2016;4:545-8.

31. Tulek F, Kahraman A, Taskin S, Yuksel S, Sertcelik A, Ortac F. Primary mucinous carcinoma of the vulva with signet ring cells deriving from the cloaca. Eur J Gynaecol Oncol. 2016;37:554-7.

32. Kaltenecker B, Manos R, McCall M, Sparzak P. Intestinal-type adenocarcinoma of the vulva: a case study. Gynecol Oncol Rep. 2019;28:133-5.

33. Lee IH, Kim MK, Lee YK, Hong SR, Lee KH. Primary mucinous adenocarcinoma of the vulva, intestinal type. Obstet Gynecol Sci. 2017;60:369-73.

34. Matsuzaki A, Saio M, Kosuge N, Aoyama H, Tamaki T, Matsumoto $\mathrm{H}$, et al. Primary villoglandular mucinous adenocarcinoma of the vulva. Case Rep Pathol. 2017;2017:1765460. 